\title{
Phenotypic characterization of polygenic type 2 diabetes in TALLYHO/JngJ mice
}

\author{
Jung Han Kim, Taryn P Stewart, Morvarid Soltani-Bejnood, Luan Wang, Jennifer M Fortuna, \\ Ola A Mostafa, Naima Moustaid-Moussa, Ahmed M Shoieb ${ }^{1}$, Michael F McEntee ${ }^{1}$, Yun Wang ${ }^{2}$, \\ Lawrence Bechtel $^{2}$ and Jürgen K Naggert ${ }^{2}$
}

Departments of Nutrition and, 'Pathobiology, The University of Tennessee, 1215 W. Cumberland Avenue, JHB 229, Knoxville, Tennessee 37996-1920, USA

${ }^{2}$ The Jackson Laboratory, Bar Harbor, Maine, USA

(Requests for offprints should be addressed to J H Kim; Email: jhkim@utk.edu)

\begin{abstract}
The TALLYHO/JngJ (TH) strain is a newly established, polygenic mouse model for type 2 diabetes (T2D) and obesity, and we have previously reported some key physiological features of this model after the overt onset of diabetes. In the present work, we conducted a comprehensive phenotypic characterization of $\mathrm{TH}$ in order to completely characterize this new and relevant model for human T2D and obesity. We monitored the development of obesity and diabetes starting at 4 weeks of age by measuring body weight, glucose tolerance, and plasma levels of insulin, glucose, and triglyceride. Additionally, histological alterations in the pancreas and glucose uptake and glucose transporter 4 (GLUT4) content in soleus muscle were also examined. Compared with age- and sex-matched C57BL/6J (B6) mice, both male and female TH mice were significantly heavier, hyperleptinemic, and hyperinsulinemic at 4 weeks of age, without glucose intolerance or hyperglycemia. TH mice maintained higher body weights throughout the study period of 16 weeks. The hyperinsulinemia in TH mice
\end{abstract}

worsened with age, but to a lesser degree in females than in males. Both the male and the female TH mice had enlarged pancreatic islets. Male TH mice showed impaired glucose tolerance at 8 weeks that became more prominent at 16 weeks. Plasma glucose levels continuously increased with age in male $\mathrm{TH}$ mice resulting in frank diabetes, while female $\mathrm{TH}$ mice remained normoglycemic throughout the study. Impaired glucose tolerance and hyperglycemia in male $\mathrm{TH}$ mice were accompanied by impaired 2-deoxyglucose uptake in the soleus muscle at basal and insulin-stimulated states, but without any reduction in GLUT4 content. Interestingly, male TH mice exhibited a drastic elevation in plasma triglyceride levels in the pre-diabetic stage that was maintained throughout the study. These findings suggest that obesity and insulin resistance are an inherent part of the TH phenotype and glucose intolerance is evident preceding progression to overt diabetes in male $\mathrm{TH}$ mice.

Journal of Endocrinology (2006) 191, 437-446

\section{Introduction}

The prevalence of diabetes is growing worldwide and it is estimated that 366 million people will be affected by the year 2030 (171 million in the year 2000; Wild et al. 2004). Diabetes is associated with an increased risk for mortality as well as morbidity. In 2002, it is estimated that 186000 deaths in US were attributed to diabetes (Hogan et al. 2003). Type 2 diabetes (T2D) is the most common form of human diabetes, accounting for approximately $90 \%$ of cases and is typically associated with obesity (Gannon 2001, Expert Committee 2003). The frequent concurrent incidence of diabetes and obesity even led one to coin the term 'diabesity', originally describing the associated symptoms of adult-onset obesity and diabetes (From the NIH 1980).

The etiology of T2D involves genetic predisposition and non-genetic risk factors such as high calorie diets and reduced physical activity (Florez et al. 2003, Leahy 2005, O'Rahilly et al. 2005). Most common forms of T2D in humans follow polygenic inheritance, i.e. multiple genes are involved in the development of the disease. Further, it is thought that T2D is genetically heterogeneous and various pathologic pathways underlie the disease in different affected individuals (Leahy 2005, O'Rahilly et al. 2005). Possibly owing to the etiological complexity, no common molecular/ cellular pathogenesis for human T2D is yet known. Understanding the pathogenesis is necessary to identify therapeutic targets as well as to generate prognostic information, which ultimately should lead to improved outcomes in affected individuals (O'Rahilly et al. 2005).

Genetic animal models have been valuable resources for T2D research, but few polygenic rodent models have been developed (Rees \& Alcolado 2005). These models include the Goto-Kakizaki (GK), the Otsuka Long-Evans Tokushima 
Fatty (OLETF) rats, the Spontaneously Diabetic Torii (SDT) rats, the Nagoya-Shibata-Yasuda (NSY) mice, the TsumuraSuzuki-Obese-Diabetes (TSOD) mice (Kim 2004), and the New Zealand Obese (NZO)/non obese non diabetic (NON) F1 hybrid mice (Leiter \& Reifsnyder 2004). Of these, the GK and SDT rats develop T2D without developing obesity and the NSY mice develop diabetes very late in life without either extreme obesity or severe hyperinsulinemia (Ueda et al. 1995, 2000, Kim 2004).

Recently, we have developed a new polygenic mouse model for T2D, TALLYHO/JngJ (TH; formerly named TallyHo), by selective breeding for hyperglycemia from an outbred colony of mice (Kim et al. 2001). TH mice exhibited obesity, hyperinsulinemia, hyperglycemia (males), and hyperlipidemia at 26 weeks of age, and genetic analysis revealed major loci and epistatic interactions accounting for the diabesity in TH mice (Kim et al. 2001). In the present study, we have conducted a more comprehensive phenotypic characterization of $\mathrm{TH}$ mice from an early age. Understanding the underlying metabolic characteristics of TH mice will provide the basis for new insights into the molecular pathogenesis of T2D in this mouse strain as well as contribute to further understanding of T2D in general.

\section{Materials and Methods}

\section{Animals}

The initial establishment of the TH strain has been detailed in our previous report (Kim et al. 2001). TH mice from the F6NE4F8, F6NE4F18 (maintained at The Jackson Laboratory, Bar Harbor, ME, USA, stock no. 005314), and the F6NE4F8 $+8 \sim+10$ (maintained at The University of Tennessee, Knoxville, TN, USA) generations were used in this study. Measurements of body weights (bw) and plasma levels of glucose, insulin, and triglyceride with age were from the F6NE4F8 and F6NE4F8 $+8 \sim+10$ generations. Histological examination, tissue glucose uptake, glucose tolerance, and plasma leptin levels were obtained from F6NE4F8+ $8 \sim+10$ mice. Since the diabetic trait in TH mice is polygenic, a simple genetic control of non-diabetic mice does not exist. Therefore, C57BL/6J (B6) inbred mice were used as arbitrary control, since B6 is one of the most commonly used strains in diabetes and obesity research and does not develop diabetes when fed standard laboratory chow. The B6 strain was also used as control in our previous study (Kim et al. 2001) and by others (Sung et al. 2005). Mice were maintained on standard rodent chow with $4 \%$ fat (5K54, LabDiet, St. Louis, MO, USA or Harlan Teklad Rodent Diet (W) 8604, Madison, WI, USA) ad libitum with free access to water ( $\mathrm{HCl}$ acidified, $\mathrm{pH} \mathrm{2 \cdot 8-3 \cdot 2)}$ under controlled temperature and humidity with a 12-h light and darkness cycle. All animal studies were carried out with the approval of either The University of Tennessee or The Jackson Laboratory Animal Care and Use Committee. Mice were euthanized by $\mathrm{CO}_{2}$ asphyxiation.
Plasma glucose, triglyceride, insulin, and leptin levels

For all studies, blood was drawn in the morning from the retro-orbital plexus via heparinized capillary tubes and plasma was obtained by centrifugation $(1200 \mathrm{~g})$ at $4{ }^{\circ} \mathrm{C}$. Plasma levels of glucose (635-100, Sigma or TR15103/1530-500, Thermo Electron, Louisville, CO, USA), and free and total glycerol (337, Sigma) were determined using commercial colorimetric assays. Plasma triglyceride concentrations were estimated by subtraction of free glycerol from total glycerol. Plasma insulin and leptin levels were determined using RIA (RI-13K, Linco Research, St. Charles, MO, USA) and ELISA kits (90030, Crystal Chem Inc., Downers Grove, IL, USA) respectively.

\section{Other blood assays}

Plasma was obtained as above and all assays were performed using a Beckman Coulter Synchron CX5 Delta chemistry analyzer (Beckman Coulter, Inc., Diagnostic Division, Brea, CA, USA). This instrument allows automated measurements of total cholesterol, HDL-cholesterol, lactate dehydrogenase, creatine kinase, alkaline phosphatase, pancreatic lipase, T4, total protein, albumin, total bilirubin, urea nitrogen, phosphorous, and calcium in the plasma.

\section{Intraperitoneal glucose tolerance test}

Mice were fasted overnight and injected intraperitoneally with glucose $(1 \mathrm{mg} / \mathrm{g}$ bw) in saline. Blood was collected via the retro-orbital plexus using a heparinized microcapillary tube at $0,15,30,60$, and $120 \mathrm{~min}$ after injection. Plasma was obtained and plasma glucose and insulin levels were determined as above.

\section{Tissue glucose uptake in vivo}

Mice were fasted overnight and injected intravenously through the tail vain with a bolus of 2-deoxy-D-glucose 1 , $2-{ }^{3} \mathrm{H}(\mathrm{N})(2-\mathrm{DG})(\mathrm{D}-4539$, Sigma; $0.5 \mu \mathrm{Ci} / \mathrm{g}$ bw) in saline either with or without insulin (I-5523, Sigma; $1 \mathrm{U} / \mathrm{ml})$. Mice were euthanized by $\mathrm{CO}_{2}$ asphyxiation $30 \mathrm{~min}$ after injection. The soleus muscle was collected, washed, blot dried, weighed, and dissolved in SOLVABLE (6NE9100, PerkinElmer, Boston, MA, USA) at room temperature. The incorporated radioactivity was counted in a scintillation counter (LS3801, Beckman, Fullerton, CA, USA). The 2-DG uptake was expressed as counts per minute divided by tissue weight (Hom et al. 1984).

\section{Histological examination}

Mice were euthanized by $\mathrm{CO}_{2}$ asphyxiation. Pancreas was dissected, fixed in 10\% neutral-buffered formalin, routinely paraffin embedded, sectioned at $3 \mu \mathrm{m}$ and the sections were stained with aldehyde fuchsin, using an orange-G 
counterstain, and hematoxylin and eosin (H\&E) for morphologic evaluation. Aldehyde fuchsin- or H\&E-stained sections were used to measure islet size using ImageJ morphometric software (Abramoff et al. 2004). All islets were photographed at $100 \times$ magnification (digital camera). Each islet in these images was manually outlined and individual islet areas (in square pixels) were recorded.

\section{Western blot analysis}

Protein extracts were prepared from the soleus muscle of B6 and $\mathrm{TH}$ mice using Tri Reagent (Sigma). Total proteins $(40 \mu \mathrm{g})$ from each sample were separated on 10\% SDS-PAGE gels and transferred to nitrocellulose using a mini trans-blot apparatus (Bio-Rad). The blots were blocked in 5\% non-fat milk dissolved in Tris-buffered saline/ $0 \cdot 1 \%$ Tween for $1 \mathrm{~h}$ at room temperature, then probed either with anti-glucose transporter 4 (GLUT4; ab654, Abcam Ltd, Cambridge, MA, USA) followed by incubation with horseradish peroxidase (HRP)-conjugated goat anti-rabbit secondary antibody (ab6721, Abcam Ltd), or with HRP-conjugated glyceraldehyde-3-phosphate dehydrogenase (GAPDH; sc-25778, Santa Cruz Biotechnology, Santa Cruz, CA, USA) for $90 \mathrm{~min}$ at room temperature. Bound antibody was visualized using the SuperSignal West Pico Chemiluminescent Substrate system (34077, Pierce Biotechnology, Rockford, IL, USA).

\section{Statistical analysis}

Data analysis was conducted by ANOVA with StatView 5.0 (Abacus Concepts, Berkeley, CA, USA). All data are presented as means \pm s.E.M.

\section{Results}

Longitudinal measurements of body weights and plasma levels of glucose, insulin, and triglyceride

At 4 weeks of age, TH mice of both sexes were significantly heavier than the age- and sex-matched B6 mice $(16 \pm 0 \cdot 4$ $(n=12)$ vs $12 \pm 1 \cdot 8(n=5), P=0 \cdot 01$, female; $18 \pm 0 \cdot 5(n=14)$ vs $15 \pm 1 \cdot 2(n=13), P=0 \cdot 01$, male; g). TH mice of both sexes were also hyperleptinemic at 4 weeks compared with B6 mice $(8.2 \pm 0.9 \quad(n=6)$, vs $2.9 \pm 0.9 \quad(n=4), P=0.003$, female; $7 \cdot 5 \pm 0 \cdot 8(n=6)$ vs $2 \cdot 3 \pm 0 \cdot 6(n=6), P=0 \cdot 0001$, male; $\mathrm{ng} / \mathrm{ml}$ ). Higher mean body weights in TH mice of both sexes were maintained throughout the study period of 16 weeks (Fig. 1). At 4 weeks, TH mice of both sexes were also hyperinsulinemic compared with B6 mice $(2 \cdot 0 \pm 1 \cdot 1 \quad(n=7)$ vs $0.9 \pm 0.8(n=6), P=0.03$, female; $2 \cdot 2 \pm 0 \cdot 9 \quad(n=6)$ vs $0 \cdot 9 \pm 0 \cdot 6(n=6), P=0 \cdot 01$, male; $\mathrm{ng} / \mathrm{ml})$. While the level of hyperinsulinemia remains relatively stable in female $\mathrm{TH}$ mice, it increased in male $\mathrm{TH}$ mice at puberty reaching their maximal levels between 8 and 12 weeks (Fig. 2A). Both prepubertal male and female $\mathrm{TH}$ mice were normoglycemic at

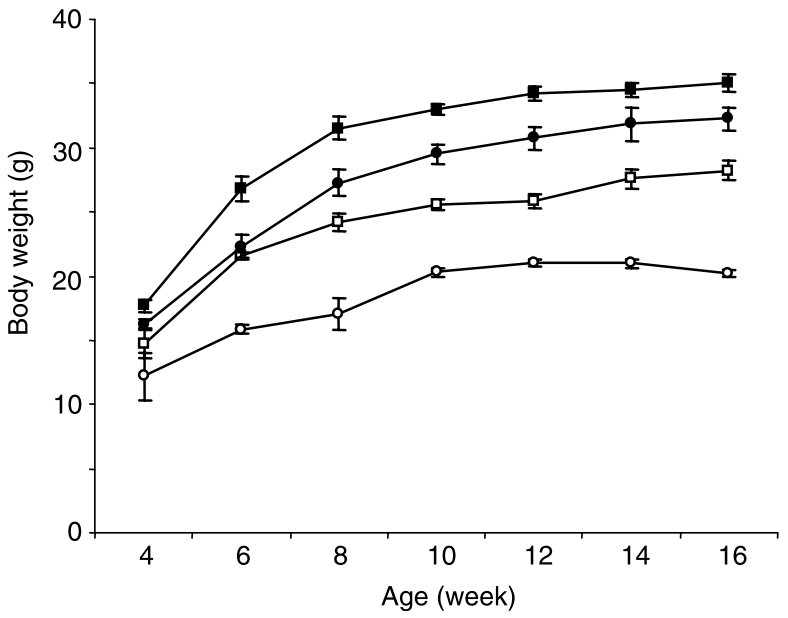

Figure 1 Body weight changes in TALLYHO/Jng (TH) and C57BL/6J (B6) mice from 4 to 16 weeks of age. Open and filled symbols represent B6 and $\mathrm{TH}$ respectively. Squares and circles represent male $(M)$ and female $(F)$ respectively. Data are means \pm s.E.M. B6-F $(n=5-13), \mathrm{B} 6-\mathrm{M}(n=7-14), \mathrm{TH}-\mathrm{F}(n=15-24)$, and TH-M $(n=12-19)$.

4 weeks (Fig. 2B). However, in post-pubertal males, plasma glucose levels continuously increased with age, reaching full-blown diabetes levels (300-400 mg/dl, non-fasting) by 14 weeks. While TH males are consistently hyperglycemic, the extent and onset of hyperglycemia varies from litter to litter. On the other hand, female $\mathrm{TH}$ mice were normoglycemic throughout the study period (Fig. 2B). Development of hypertriglyceridemia was also notable in TH mice; male TH mice exhibited a striking rise in plasma triglyceride levels between 6 and 10 weeks and maintained high levels throughout the study (Fig. 2C). However, female TH mice did not exhibit the triglyceride spike as shown in TH males, although they were hypertriglyceridemic compared with B6 mice (Fig. 2C).

We also measured other blood parameters to determine additional metabolic differences between $\mathrm{TH}$ and $\mathrm{B} 6$ mice (Table 1). Compared with male B6 mice, male TH mice were hypercholesterolemic. In addition, notable were significantly lower activity of pancreatic lipase and alkaline phosphatase, and lower levels of T4, total bilirubin, and urea nitrogen in male TH compared with B6 mice (Table 1); however, these were within normal ranges for male mice (unpublished The Jackson Laboratory reference data for retired breeders from strains $\mathrm{B} 6, \mathrm{C} 3 \mathrm{H} / \mathrm{HeJ}$, and $\mathrm{BALB} / \mathrm{cJ})$.

\section{Histology of pancreatic islets}

At both pre- (4 weeks) and post-diabetic (16 weeks) stages, the mean size of the isles was significantly larger in $\mathrm{TH}$ mice than in age- and sex-matched B6 mice, as measured by morphometry (Fig. 3). This suggests the presence of continuous potent stimuli for insulin secretion in $\mathrm{TH}$ mice. H\&E-stained pancreatic islets were also examined for 

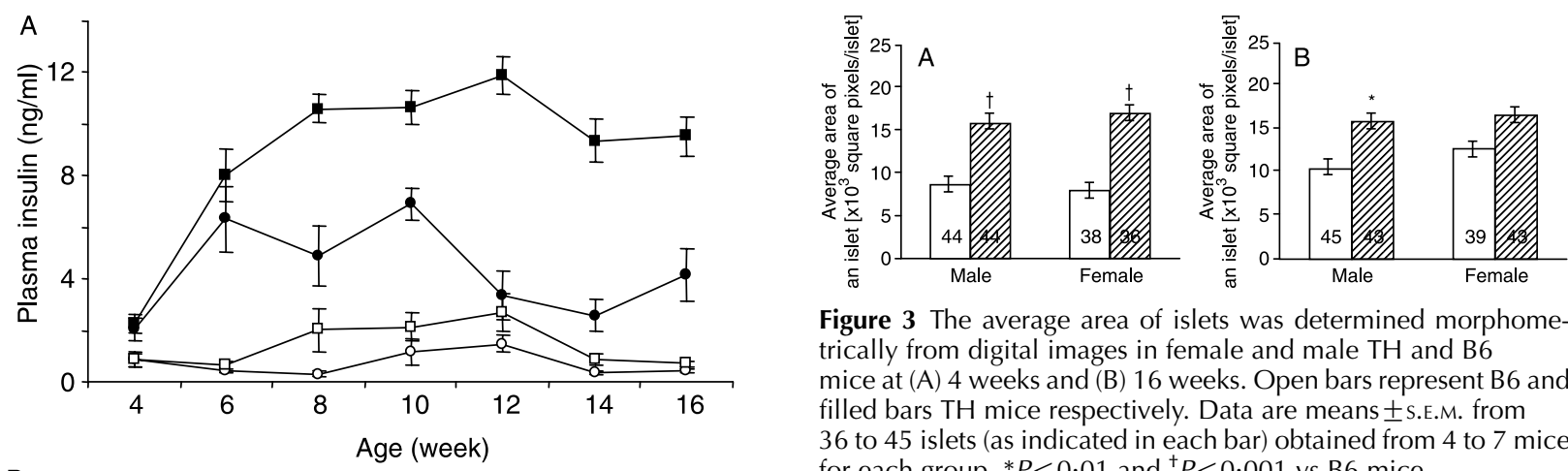

Figure 3 The average area of islets was determined morphometrically from digital images in female and male $\mathrm{TH}$ and $\mathrm{B} 6$ mice at (A) 4 weeks and (B) 16 weeks. Open bars represent B6 and filled bars TH mice respectively. Data are means \pm S.E.M. from 36 to 45 islets (as indicated in each bar) obtained from 4 to 7 mice

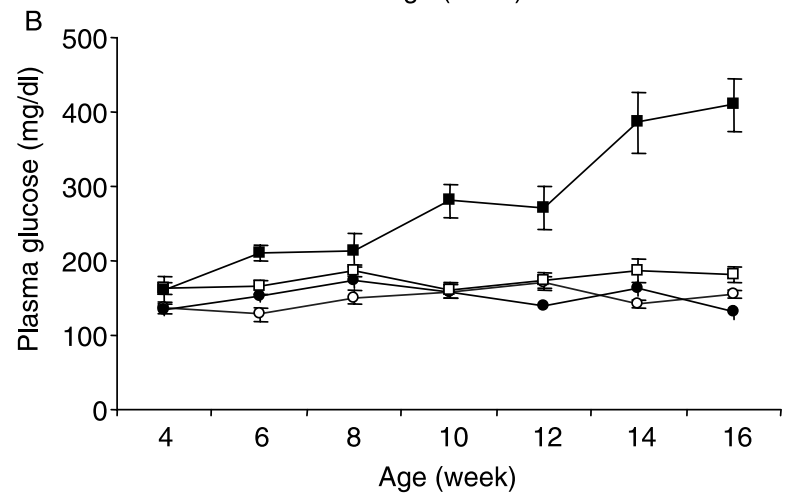
for each group. ${ }^{*} P<0.01$ and ${ }^{\dagger} P<0.001$ vs B6 mice.

\section{Intraperitoneal glucose tolerance test (IPGTT)}

Male TH mice were glucose tolerant at 4 weeks of age, but exhibited impaired glucose tolerance at 8 weeks that further deteriorated at 16 weeks (Fig. 5A). As glucose intolerance can be attributable to insulin resistance and/or defects in insulin secretion, we measured the changes in plasma insulin levels during IPGTT to evaluate the contribution of insulin secretory defects. At 8 weeks of age, fasting and post-glucose challenge insulin levels were significantly higher in male $\mathrm{TH}$ mice than B6 males (Fig. 5A), indicating presence of insulin resistance in TH. At 16 weeks of age, the fasting insulin levels were higher in TH males, but total insulin secretion in response to glucose, as assessed by the area under the curve of insulin in IPGTT, was not significantly different between TH and $\mathrm{B} 6$ mice (Fig. 5A). On the other hand, female TH mice showed normal glucose tolerance at all ages determined (Fig. 5B). However, their fasting and post-glucose challenge insulin levels were significantly higher than those of B6 mice at 8 and 16 weeks of age (Fig. 5B).

\section{Glucose uptake}

Glucose uptake, the first step of glucose metabolism in the body was tested in vivo in the insulin sensitive soleus muscle of male TH and B6 mice, using 2-DG. TH mice exhibited significant reductions in both basal and insulin-stimulated 2-DG uptake compared with the age- and sex-matched B6 mice (Fig. 6).

\section{GLUT4 content in soleus muscle}

histologic abnormalities. Islet cells in 4-week-old TH males, 16-week-old TH females, and 16-week-old B6 males were all morphologically similar with relatively abundant and uniform cytoplasmic granulation and scattered mitoses, particularly in younger mice (Fig. 4). In contrast, islets in 16 week-old TH males often had evidence of degeneration characterized by cells with reduced amounts of cytoplasm, granulation (often sequestered in a perinuclear location), occasional vacuolation, scattered apoptotic and/or necrotic cells and, in some instances, separation of cells by a fine eosinophilic stroma (mild fibrosis).

GLUT4 mediates the rate-limiting step of insulin-responsive glucose transport in skeletal muscle (Bell et al. 1990). The abundance of GLUT4 protein in soleus muscle was not significantly different between B6 and TH mice (Fig. 7).

\section{Discussion}

Previously, we reported the physiological features of TH mice at 26 weeks of age after the overt onset of diabetes (Kim et al. 
Table 1 Blood parameters in 8 week-old C57BL/6J and TALLYHO/JngJ mice (males, non fasting). Values are means \pm s.E.M.

Total cholesterol $(\mathrm{mg} / \mathrm{dl})$ HDL-cholesterol (mg/dl) Lactate dehydrogenase (IU/I) Creatine kinase (IU/I) Alkaline phosphatase (IU/I) Pancreatic lipase (U/I) $\mathrm{T} 4(\mu \mathrm{g} / \mathrm{dl})$ Total protein $(\mathrm{g} / \mathrm{dl})$ Albumin (g/dl) Total bilirubin $(\mathrm{mg} / \mathrm{dl})$ Urea nitrogen $(\mathrm{mg} / \mathrm{dl})$ Phosphorus (mg/dl) Calcium $(\mathrm{mg} / \mathrm{dl})$

\begin{tabular}{|c|c|c|}
\hline C57BL/6J $(n=10)$ & TALLYHO/JngJ $(n=6)$ & $P$ value \\
\hline $99 \cdot 7 \pm 1 \cdot 7$ & $144 \cdot 7 \pm 8 \cdot 6$ & $<0 \cdot 0001$ \\
\hline $69 \cdot 8 \pm 1 \cdot 6$ & $105 \cdot 9 \pm 7 \cdot 8$ & $<0 \cdot 0001$ \\
\hline $585 \cdot 9 \pm 197 \cdot 7$ & $494 \cdot 0 \pm 154 \cdot 3$ & $0 \cdot 73$ \\
\hline $2056 \pm 968$ & $1842 \pm 1086$ & $0 \cdot 9$ \\
\hline $174 \pm 12$ & $127 \pm 6$ & $0 \cdot 01$ \\
\hline $123 \pm 8$ & $65 \pm 1$ & $<0 \cdot 0001$ \\
\hline $7 \cdot 57 \pm 0 \cdot 38$ & $4 \cdot 70 \pm 0 \cdot 47$ & $0 \cdot 0004$ \\
\hline $5 \cdot 7 \pm 0 \cdot 34$ & $6 \cdot 0 \pm 0 \cdot 24$ & $0 \cdot 19$ \\
\hline $2 \cdot 9 \pm 0 \cdot 14$ & $2 \cdot 5 \pm 0 \cdot 06$ & $0 \cdot 06$ \\
\hline $0 \cdot 8 \pm 0 \cdot 05$ & $0 \cdot 6 \pm 0 \cdot 11$ & $0 \cdot 04$ \\
\hline $30 \cdot 9 \pm 0 \cdot 9$ & $24 \cdot 2 \pm 1 \cdot 2$ & $0 \cdot 0006$ \\
\hline $8 \cdot 3 \pm 0 \cdot 3$ & $8 \cdot 1 \pm 0 \cdot 5$ & $0 \cdot 75$ \\
\hline $10 \cdot 3 \pm 0 \cdot 2$ & $10 \cdot 6 \pm 0 \cdot 2$ & $0 \cdot 1$ \\
\hline
\end{tabular}
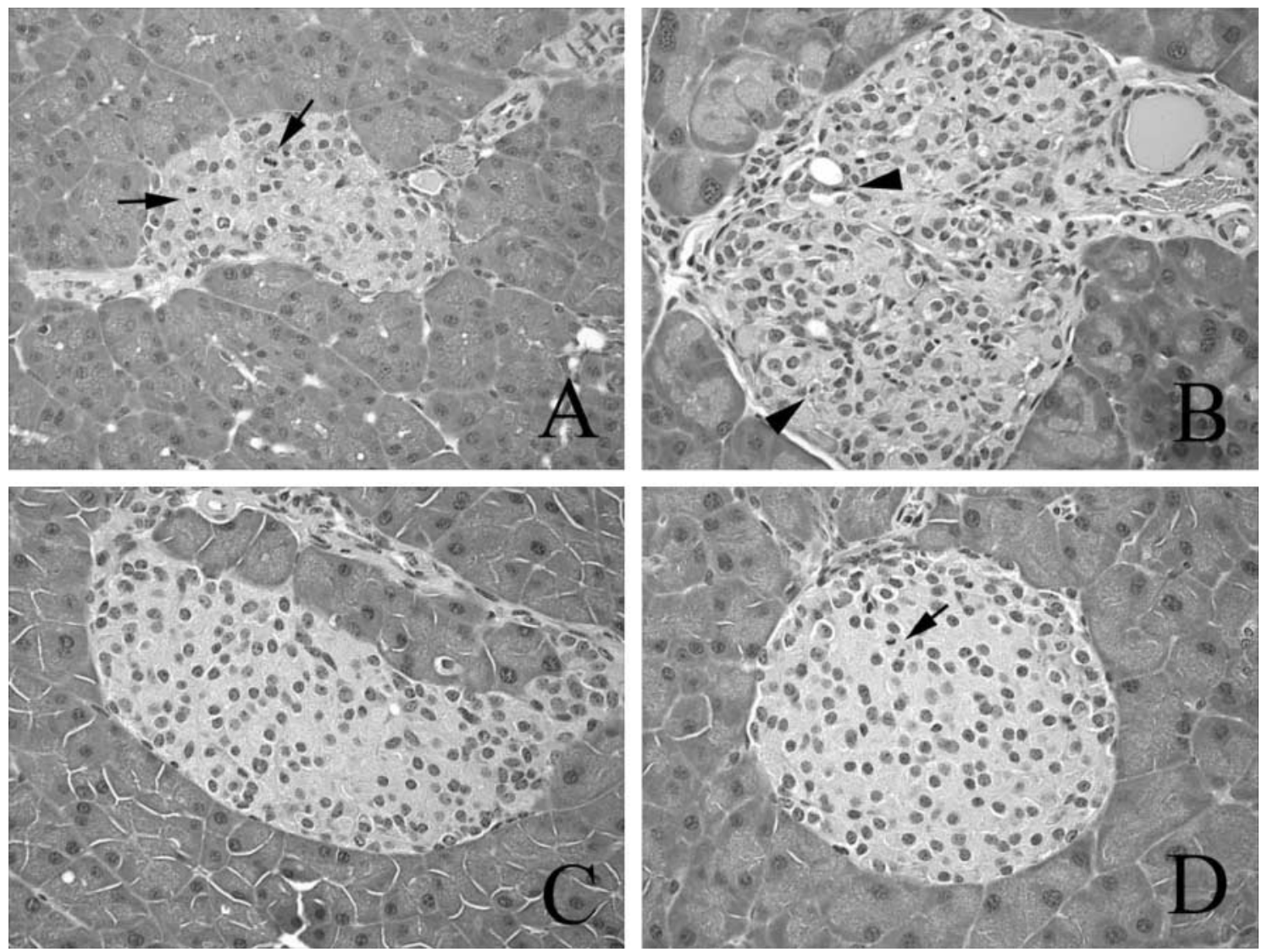

Figure 4 Representative photomicrographs of islets in H\&E-stained sections from (A) 4-week-old TH male, (B) 16-week-old TH male, (C) 16-week-old TH female, and (D) 16-week-old B6 male mice. Islet cells in all but the 16-week-old TH males contained granulated cytoplasm, whereas the older TH male islets contained smaller cells with less cytoplasmic granulation, scattered apoptotic or necrotic cells (arrow heads), and occasional evidence of fibrosis. Mitotic activity (arrows) was most evident in younger mice and not apparent in older TH males (all images $400 \times$ original magnification). 
2001). In the present study, we conducted a more comprehensive characterization from an early age.

Normal blood enzyme levels indicate that no major organ damage is evident at the 8 -weeks time point. However, plasma pancreatic lipase activity was significantly lower in $\mathrm{TH}$ male mice than in B6 controls. It is interesting to note that $\mathrm{TH}$ mice also have significantly lower gene expression of pancreatic lipase related protein 1 (Brown et al. 2005), a secreted protein that can function as a lipase (Grusby et al. 1990). Whether this gene expression difference can account for the reduced lipase levels observed in the plasma remains to be determined. It is also noted that plasma thyroid hormone (T4) levels were found to be significantly lower in $\mathrm{TH}$ than in B6 mice, perhaps indicative of a lower metabolic rate in $\mathrm{TH}$
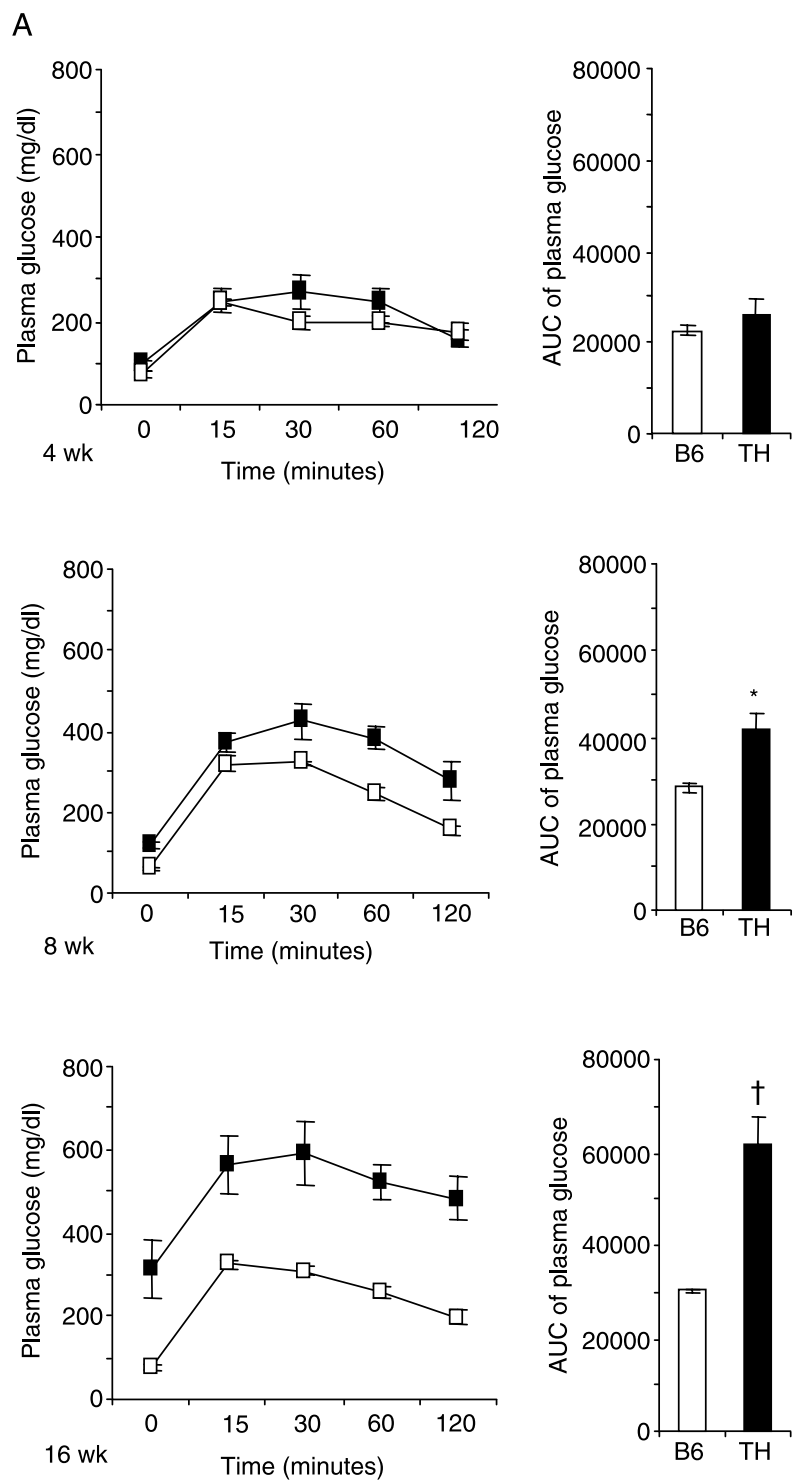

mice (Sane \& Taskinen 1993). It has also recently been shown that hypothyroidism is associated with decreased peripheral glucose utilization in rats (Cettour-Rose et al. 2005).

For both male and female $\mathrm{TH}$ mice, the metabolic aberrations shown at weaning age included increased mean body weights and plasma insulin levels (Figs 1 and 2A). TH mice were also hyperleptinemic at this age. Since plasma leptin levels are positively correlated with adiposity (Leibel 2002) and hyperinsulinemia reflects pancreatic attempts to overcome peripheral insulin resistance (Lebovitz 2001), obesity and reduced sensitivity to insulin action appear to be primary features in $\mathrm{TH}$ mice. However, the obesity in $\mathrm{TH}$ mice is not as severe as that in some other polygenic T2D models including NZO (43 g, male, 8 weeks) (Leiter \&
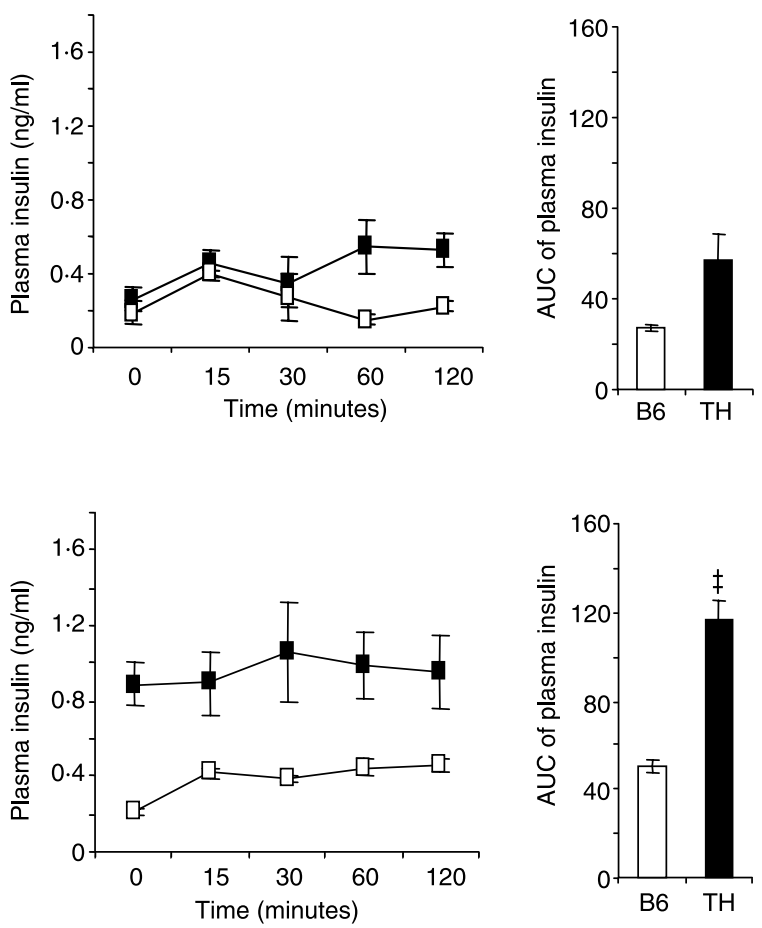

Figure 5 (continued)
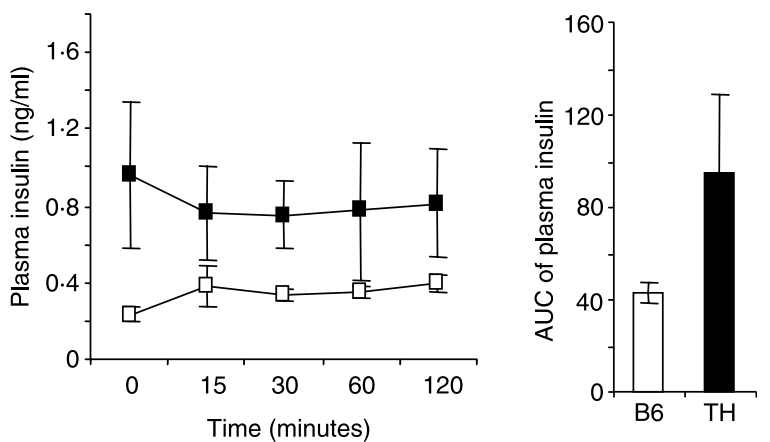

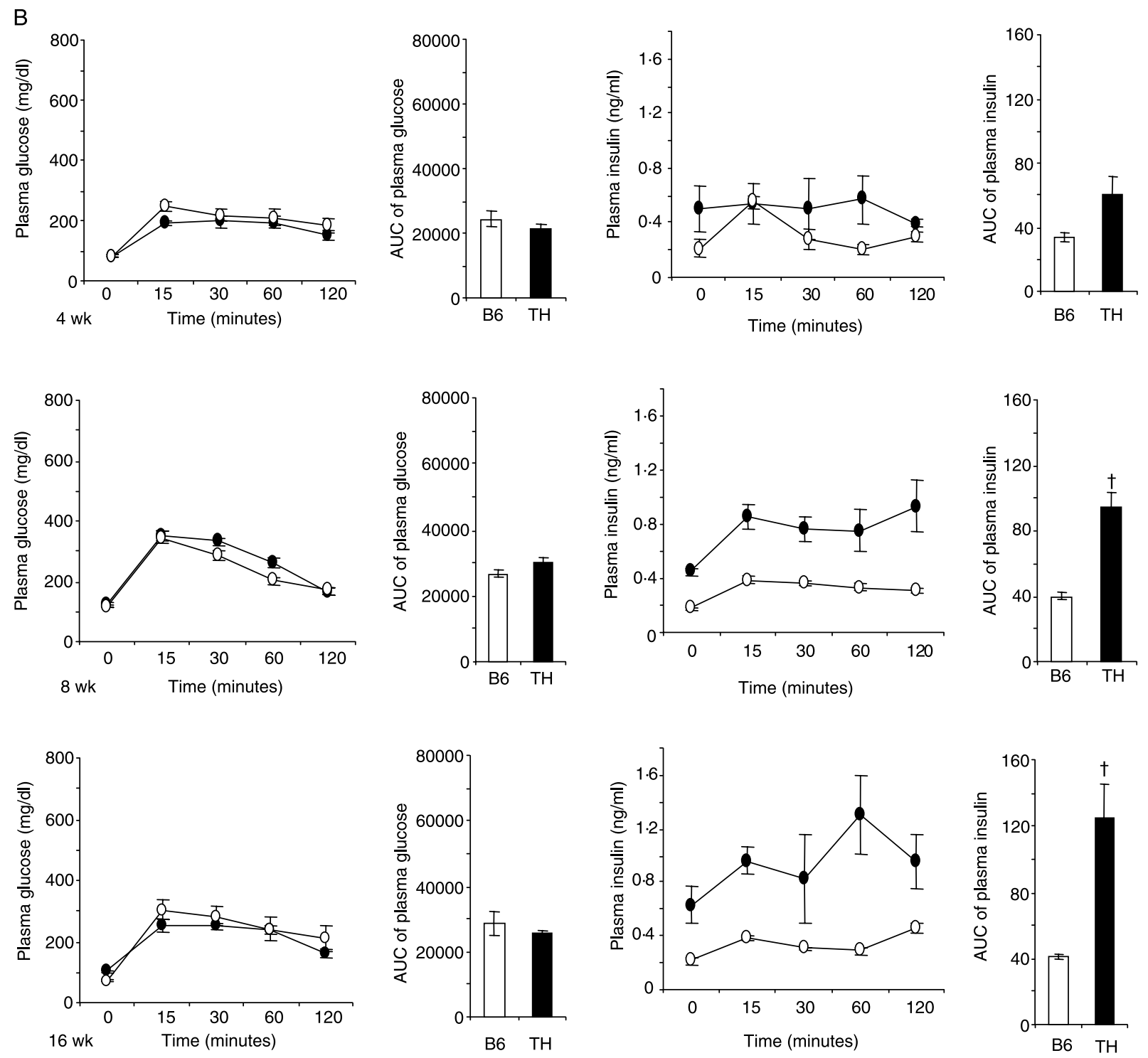

Figure 5 Intraperitoneal glucose tolerance (IPGTT) in (A) male and (B) female TH and B6 mice at 4, 8, and 16 weeks of age $(n=5$ for each group). Mice were fasted overnight and injected intraperitoneally with a bolus of glucose (1 mg/g body weight) in saline. Blood was obtained at times $0,15,30,60$, and $120 \mathrm{~min}$ after injection, and plasma glucose and insulin levels were measured. Area under the curve (AUC) of glucose and insulin levels during the IPGTT was also calculated. Open and filled symbols represent B6 and TH respectively. Data are means \pm S.E.M. ${ }^{*} P<0 \cdot 01,{ }^{\dagger} P<0 \cdot 001$, and ${ }^{\ddagger} P<0 \cdot 0001$ vs B6 mice.

Reifsnyder 2004) and TSOD (45 g, male, 8 weeks) mice (Suzuki et al. 1999).

The hyperinsulinemia in $\mathrm{TH}$ mice was accompanied by hypertrophied islets, possibly reflecting the $\beta$-cell adaptation to the increased loads caused by the body's insulin resistance (Fig. 3). With disease progression in TH males, there was also histologic evidence of islet degeneration (Fig. 4) presumably reflecting chronic over-stimulation of $\beta$-cells, although $\beta$-cell mass was adequate to maintain elevated levels of circulating insulin. The cellular pathobiology responsible for this change and characterization of further deterioration with age remains to be determined.

Impaired glucose tolerance is well recognized as a precursor to the development of T2D in human populations including Pima Indians, in which subjects with impaired glucose tolerance are at a higher risk than those with normal tolerance (Knowler et al. 1990). The progress from glucose intolerance to diabetes is known to be accompanied by failure of insulin secretion in response to glucose (Knowler et al. 1990, DeFronzo 1997, Lebovitz 2001). The high risk of developing 


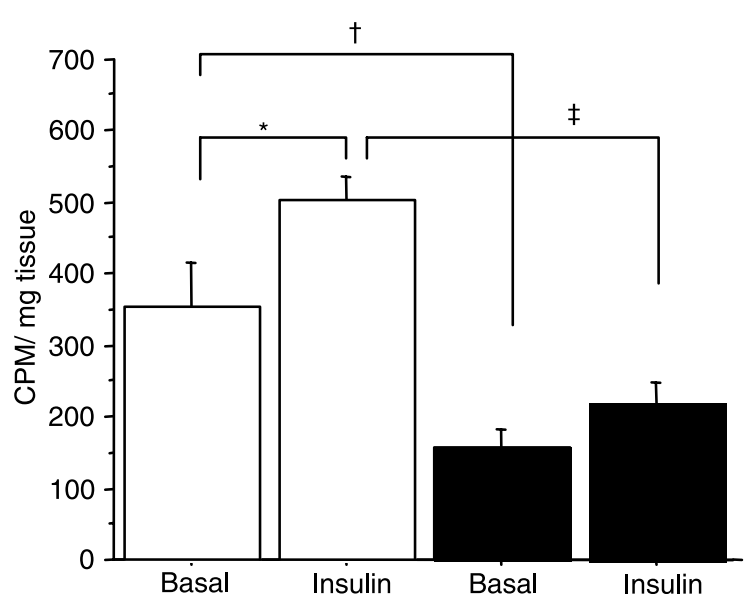

Figure 6 In vivo 2-deoxy-D-glucose 1, $2-{ }^{3} \mathrm{H}(\mathrm{N})$ (2-DG) uptake in the soleus muscles of TH and B6 mice at 6 weeks of age (males). Mice were fasted overnight and injected intravenously through the tail vain with a bolus of 2 -DG $(0.5 \mu \mathrm{Ci} / \mathrm{g}$ body weight $)$ in saline without (basal) or with insulin (insulin) $(1 \mathrm{U} / \mathrm{ml})$, and tissue was harvested $30 \mathrm{~min}$ after injection. The 2-DG uptake was expressed as counts per minute (CPM) normalized by tissue weight (mg). Open bars represent B6 (basal, $n=6$; insulin, $n=6$ ) and closed bars represent TH mice (basal, $n=5$; insulin, $n=6$ ) respectively. Data are means \pm S.E.M. ${ }^{*} P=0 \cdot 02,{ }^{\dagger} P=0 \cdot 004$, and ${ }^{\ddagger} P=0 \cdot 0001$.

T2D correlates with reduced $\beta$-cell function relative to the degree of insulin sensitivity (Kahn et al. 1993, 2001). Early alterations in both insulin sensitivity and secretion were observed in individuals, who progress from normal glucose tolerance to impaired glucose tolerance in a longitudinal study of a Pima Indian population (Weyer et al. 1999). We found that glucose intolerance was accompanied by increased insulin secretion during IPGTT in male TH mice at 8 weeks

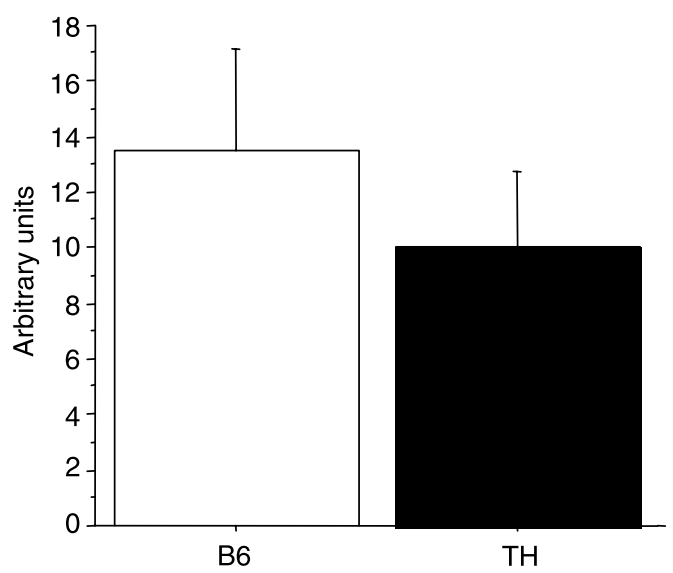

Figure 7 Western blot analysis for glucose transporter 4 (GLUT4) in the soleus muscles of TH and B6 mice at 6-10 weeks of age (males, $n=4$ for each group). Mean GLUT4 content of soleus muscle homogenates determined by densitometric analysis of autoradiographs are displayed as arbitrary units after normalization to glyceraldehyde-3-phosphate dehydrogenase (GAPDH) content levels in the same blot. Data are mean \pm s.E.M. of age (Fig. 5A), possibly via a compensatory hypersecretion mechanism in response to diminished insulin action. However, this compensatory increase of insulin secretion during IPGTT was not profound in TH males at 16 weeks of age when their glucose intolerance became severe (Fig. 5A). Therefore, it appears that insulin resistance is a primary defect of $\mathrm{TH}$ mice and with disease progression insufficient $\beta$-cell compensation leads to glucose intolerance and diabetes in male TH mice. Sung et al. (2005) recently reported mild glucose intolerance, reduced insulin secretion during IPGTT, and elevated leptin expression in male TH mice at 4 weeks of age with little sign of insulin resistance. These results are contradictory to our observations, but higher dose of glucose load $(2 \mathrm{~g} / \mathrm{kg}$ bw) in IPGTT for their study could possibly explain the glucose intolerance. Sung et al. (2005) have proposed that hyperleptinemia may cause the inhibition of insulin secretion in TH mice via direct effects of leptin on the pancreas. However, in our study, there was no evidence of insulin secretion defects during IPGTT in TH mice at 4 weeks of age, despite their hyperleptinemia. In addition, Sung et al. (2005) did not observe obesity, hyperinsulinemia, or hypertriglyceridemia in $\mathrm{TH}$ mice compared with $\mathrm{B} 6$ at a young age, unlike in our study. Presently, it is unknown what causes these discrepancies in the observations. It is conceivable that environmental factors may be involved in different phenotypic expressions in $\mathrm{TH}$ mice at different laboratories, but this remains to be confirmed.

Sex dimorphism for diabetes is commonly observed in T2D rodent models including NSY, TSOD, Lepr ${ }^{d b}$, and Lep ${ }^{o b}$ mice, and in OLETF rats (Leiter \& Chapman 1994, Hirayama et al. 1999, Rees \& Alcolado 2005). In Lepr ${ }^{d b}$ and Lep ${ }^{o b}$ mutant mice, the protection from diabetes in female mice can be attributed to low hepatic estrogen sulfotransferase activity, which prevents virilization of liver metabolism (Leiter \& Chapman 1994). It has also been demonstrated that estrogen increases the density of insulin receptor in the hepatocyte membrane (Krakower et al. 1993), perhaps offsetting postreceptor insulin resistance in female rodents.

In support of glucose intolerance and hyperglycemia in male TH mice, soleus muscle in TH males exhibited lower basal and insulin-stimulated 2-DG uptake than in B6 mice even at a young age of 6 weeks (Fig. 6), stressing insulin resistance as a primary defect of TH mice. Reduced glucose uptake in skeletal muscle has also previously been reported in other animal models of obesity and T2D, including NZO mice (Veroni et al. 1991) and OLETF rats (Sato et al. 1995). In addition to skeletal muscle, a classic insulin target is adipose tissue (Biddinger \& Kahn 2006). Indeed, insulin insensitivity on glucose uptake in adipocytes from diabetic humans as well as animals has been demonstrated (Hjollund et al. 1985, Ikeda 1994). Previously, we reported a reduced insulin-stimulating glucose uptake in adipose tissue of congenic mice carrying an obesity quantitative trait locus (tabw2) derived from TH mice (Kim et al. 2005). Congenic mice represent a simplified system that allows one to tease out polygenic traits (Lyons et al. 2000). Like the tabw2 congenic mice, the parental TH mice might have diminished glucose 
uptake in adipose tissue, but this remains to be tested. In addition, future study is needed to identify the major tissues that are defective in $\mathrm{TH}$ mice, in order to understand the causative mechanism(s) underlying T2D in this model.

A major mechanism accounting for impaired glucose uptake in skeletal muscle is decreased GLUT4 content and/or its altered translocation to the plasma membrane (Angel et al. 1996, Kelley et al. 1996, Toide et al. 1997, Miura et al. 2001). Although significantly suppressed GLUT4 expression was exhibited in skeletal muscle of insulin resistant rodent models (Kahn \& Pedersen 1993, Machado et al. 1993), there was no evidence of reduced GLUT4 content in soleus muscle from TH male mice (Fig. 7). GLUT4 protein is usually sequestered into specialized vesicles within the cell at basal conditions and translocates to the plasma membrane following activation of insulin signal transduction when post-prandial glucose levels rise (Watson \& Pessin 2006). Blunted GLUT4 translocation to the plasma membrane from the intracellular storage vesicles has been reported in insulin resistant states (Shao et al. 2000, Miura et al. 2001). Since B6 and TH mice exhibit comparable GLUT4 levels, it is possible that GLUT4 translocation is the defective step in glucose transport in TH mice.

Insulin resistance was profoundly associated with hypertriglyceridemia in male $\mathrm{TH}$ mice (Fig. 2C). Roden et al. (1996) demonstrated that an i.v. infusion of a triglyceride emulsion into healthy individuals decreased muscle glycogen synthesis by $50 \%$ and the rate of whole-body glucose uptake by $46 \%$. Dresner et al. (1999) also demonstrated that lipid infusion abolished insulin signaling. Family studies indicate that patients with hypertriglyceridemia are at increased risk of developing T2D and hypertriglyceridemia served as a risk marker of glucose intolerance and T2D in such families (Sane \& Taskinen 1993). Normalization of triglyceride levels lowered fasting plasma glucose levels more than three times and improved insulin sensitivity in subjects with extreme hypertriglyceridemia and overt diabetes (Mingrone et al. 1999). It may be speculated that the severe hypertriglyceridemia preceding the overt hyperglycemia might contribute to the development of diabetes in male TH mice, but this remains to be tested.

In summary, this study reveals that obesity and insulin resistance emerge early in the development of $\mathrm{T} 2 \mathrm{D}$ in $\mathrm{TH}$ mice. Severe hypertriglyceridemia and impaired glucose tolerance precede overt hyperglycemia in male $\mathrm{TH}$ mice. The impaired glucose tolerance and hyperglycemia are in part attributed to reduced glucose uptake in muscle. This new mouse model recapitulates many of the metabolic abnormalities observed in human T2D and will be a valuable tool for identification of the underlying molecular defects.

\section{Acknowledgements}

This work was supported by grants from the American Diabetes Association (7-04-RA-52), the American Heart Association $(0235345 \mathrm{~N})$, and the Center of Genomics and Bioinformatics ( $\mathrm{H} \mathrm{K}$ ), National Institutes of Health
(DK-46977 and DK-071994, J K N; RR-16645, M F M), and the TN Agricultural Experiment Station (N M-M). The Jackson Laboratory core services were supported by an institutional grant (CA-24190). We thank Cindy S Avery and Son Yong Karst for assistance with animal care. We thank Dr Edward H Leiter for the valuable review of the manuscript. The authors declare that there is no conflict of interest that would prejudice the impartiality of this scientific work.

\section{References}

Abramoff MD, Magelhaes PJ \& Ram SJ 2004 Image processing with imageJ. Biophotonics International 11 36-42.

Angel I, Burcelin R, Prouteau M, Girard J \& Langer SZ 1996 Normalization of insulin secretion by a selective alpha 2 -adrenoceptor antagonist restores GLUT-4 glucose transporter expression in adipose tissue of type II diabetic rats. Endocrinology 137 2022-2027.

Bell GI, Kayano T, Buse JB, Burant CF, Takeda J, Lin D, Fukumoto H \& Seino S 1990 Molecular biology of mammalian glucose transporters. Diabetes Care $\mathbf{1 3}$ 198-208.

Biddinger SB \& Kahn CR 2006 From mice to men: insights into the insulin resistance syndromes. Annual Review of Physiology 68 123-158.

Brown AC, Olver W, Donnelly C, May M, Naggert JK, Shaffer DJ \& Roopenian DC 2005 Searching QTLs by gene expression: analysis of diabesity. BioMed Central Genetics 612.

Cettour-Rose P, Theander-Carillo C, Asensio C, Klein M, Visser TJ, Burger AG, Meier CA \& Rohner-Jeanrenaud F 2005 Hypothyroidism in rats decreases peripheral glucose utilization, a defect partially corrected by central leptin infusion. Diabetologia 48 624-633.

DeFronzo RA 1997 Insulin resistance: a multifaceted syndrome responsible for NIDDM, obesity, hypertension, dyslipidaemia and atherosclerosis. Netherlands Journal of Medicine 50 191-197.

Dresner A, Laurent D, Marcucci M, Griffin ME, Dufour S, Cline GW, Slezak LA, Andersen DK, Hundal RS, Rothman DL et al. 1999 Effects of free fatty acids on glucose transport and IRS-1-associated phosphatidylinositol 3-kinase activity. Journal of Clinical Investigation 103 253-259.

Expert Committee on the Diagnosis and Classification of Diabetes Mellitus 2003 Report of the expert committee on the diagnosis and classification of diabetes mellitus. Diabetes Care 26 S5-S20.

Florez JC, Hirschhorn J \& Altshuler D 2003 The inherited basis of diabetes mellitus: implications for the genetic analysis of complex traits. Annual Review of Genomics and Human Genetics 4 257-291.

From the NIH 1980 Successful diet and exercise therapy is conducted in Vermont for 'diabesity'. Journal of the American Medical Association 243 519-520.

Gannon M 2001 Molecular genetic analysis of diabetes in mice. Trends in Genetics 17 S23-S28.

Grusby MJ, Nabavi N, Wong H, Dick RF, Bluestone JA, Schotz MC \& Glimcher LH 1990 Cloning of an interleukin-4 inducible gene from cytotoxic T lymphocytes and its identification as a lipase. Cell 60 451-459.

Hirayama I, Yi Z, Izumi S, Arrai I, Suzuki W, Nagamachi Y, Kuwano H, Takeuchi T \& Izumi T 1999 Genetic analysis of obese diabetes in the TSOD mouse. Diabetes 48 1183-1191.

Hjollund E, Beck-Nielsen H, Pedersen O, Richelsen B \& Sorensen NS 1985 Defective non-insulin-mediated and insulin-mediated glucose transport and metabolism in adipocytes from obese and lean patients with untreated type 2 diabetes mellitus. Diabetic Medicine 2 468-473.

Hogan P, Dall T, Nikolov P \& American Diabetes Association 2003 Economic costs of diabetes in the US in 2002. Diabetes Care 26 917-932.

Hom FG, Goodner CJ \& Berrie MA 1984 A [ $\left.{ }^{3} \mathrm{H}\right] 2$-deoxyglucose method for comparing rates of glucose metabolism and insulin responses among rat tissues in vivo. Validation of the model and the absence of an insulin effect on brain. Diabetes 33 141-152.

Ikeda H 1994 KK mouse. Diabetes Research and Clinical Practice 24( Suppl 1) S313-S316. 
Kahn BB \& Pedersen O 1993 Suppression of GLUT4 expression in skeletal muscle of rats that are obese from high fat feeding but not from high carbohydrate feeding or genetic obesity. Endocrinology 132 13-22.

Kahn SE, Prigeon RL, McCulloch DK, Boyko EJ, Bergman RN, Schwartz MW, Neifing JL, Ward WK, Beard JC, Palmer JP et al. 1993 Quantification of the relationship between insulin sensitivity and beta-cell function in human subjects. Evidence for a hyperbolic function. Diabetes $\mathbf{4 2}$ 1663-1672.

Kahn SE, Prigeon RL, Schwartz RS, Fujimoto WY, Knopp RH, Brunzell JD \& Porte D Jr. 2001 Obesity, body fat distribution, insulin sensitivity and islet beta-cell function as explanations for metabolic diversity. Journal of Nutrition 131 354S-360S.

Kelley DE, Mintun MA, Watkins SC, Simoneau JA, Jadali F, Fredrickson A, Beattie J \& Theriault R 1996 The effect of non-insulin-dependent diabetes mellitus and obesity on glucose transport and phosphorylation in skeletal muscle. Journal of Clinical Investigation 97 2705-2713.

Kim JH 2004 Quantitative trait loci for obesity and type 2 diabetes in rodents. In Genomics and Proteomics in Nutrition, pp 15-48. Eds C Berdanier \& N Moustaid-Moussa. New York: Marcel Dekker.

Kim JH, Sen S, Avery CS, Simpson E, Chandler P, Nishina PM, Churchill GA \& Naggert JK 2001 Genetic analysis of a new mouse model for noninsulin-dependent diabetes. Genomics 74 273-286.

Kim JH, Stewart TP, Zhang W, Kim HY, Nishina PM \& Naggert JK 2005 Type 2 diabetes mouse model TallyHo carries an obesity gene on chromosome 6 that exaggerates dietary obesity. Physiological Genomics 22 171-181.

Knowler WC, Pettitt DJ, Saad MF \& Bennett PH 1990 Diabetes mellitus in the Pima Indians: incidence, risk factors and pathogenesis. Diabetes/Metabolism Reviews 6 1-27.

Krakower GR, Meier DA \& Kissebah AH 1993 Female sex hormones, perinatal, and peripubertal androgenization on hepatocyte insulin dynamics in rats. American Journal of Physiology 264 E342-E347.

Leahy JL 2005 Pathogenesis of type 2 diabetes mellitus. Archives of Medical Research 36 197-209.

Lebovitz HE 2001 Diagnosis, classification, and pathogenesis of diabetes mellitus. Journal of Clinical Psychiatry 62 5-9.

Leibel RL 2002 The role of leptin in the control of body weight. Nutrition Reviews 60 S15-S19.

Leiter EH \& Chapman HD 1994 Obesity-induced diabetes (diabesity) in C57BL/KsJ mice produces aberrant trans-regulation of sex steroid sulfotransferase genes. Journal of Clinical Investigation 93 2007-2013.

Leiter EH \& Reifsnyder PC 2004 Differential levels of diabetogenic stress in two new mouse models of obesity and type 2 diabetes. Diabetes $\mathbf{5 3}$ S4-S11.

Lyons PA, Armitage N, Argentina F, Denny P, Hill NJ, Lord CJ \& Wilusz MB 2000 Congenic mapping of the type 1 diabetes locus, Idd3, to a $780-\mathrm{kb}$ region of mouse chromosome 3: identification of a candidate segment of ancestral DNA by haplotype mapping. Genome Research 10 446-453.

Machado UF, Shimizu Y \& Saito M 1993 Decreased glucose transporter (GLUT 4) content in insulin-sensitive tissues of obese aurothioglucose- and monosodium glutamate-treated mice. Hormone and Metabolic Research 25 462-465.

Mingrone G, Henriksen FL, Greco AV, Krogh LN, Capristo E, Gastaldelli A, Castagneto M, Ferrannini E, Gasbarrini G \& Beck-Nielsen H 1999 Triglyceride-induced diabetes associated with familial lipoprotein lipase deficiency. Diabetes 48 1258-1263.
Miura T, Suzuki W, Ishihara E, Arai I, Ishida H, Seino Y \& Tanigawa K 2001 Impairment of insulin-stimulated GLUT4 translocation in skeletal muscle and adipose tissue in the Tsumura Suzuki obese diabetic mouse: a new genetic animal model of type 2 diabetes. European Journal of Endocrinology 145 785-790.

O'Rahilly S, Barroso I \& Wareham NJ 2005 Genetic factors in type 2 diabetes: the end of the beginning? Science 307 370-373.

Rees DA \& Alcolado JC 2005 Animal models of diabetes mellitus. Diabetic Medicine 22 359-370.

Roden M, Price TB, Perseghin G, Petersen KF, Rothman DL, Cline GW \& Shulman GI 1996 Mechanism of free fatty acid-induced insulin resistance in humans. Journal of Clinical Investigation 97 2859-2865.

Sane T \& Taskinen MR 1993 Does familial hypertriglyceridemia predispose to NIDDM? Diabetes Care 16 1494-1501.

Sato T, Asahi Y, Toide K \& Nakayama N 1995 Insulin resistance in skeletal muscle of the male Otsuka Long-Evans Tokushima Fatty rat, a new model of NIDDM. Diabetologia 38 1033-1041.

Shao J, Yamashita H, Qiao L \& Friedman JE 2000 Decreased Akt kinase activity and insulin resistance in C57BL/KsJ-Leprdb/db mice. Journal of Endocrinology 167 107-115.

Sung YY, Lee YS, Jung WH, Kim HY, Cheon HG, Yang SD \& Rhee SD 2005 Glucose intolerance in young TallyHo mice is induced by leptinmediated inhibition of insulin secretion. Biochemical and Biophysical Research Communications 338 1779-1787.

Suzuki W, Iizuka S, Tabuchi M, Funo S, Yanagisawa T, Kimura M, Sato T, Endo T \& Kawamura H 1999 A new mouse model of spontaneous diabetes derived from ddY strain. Experimental Animals 48 181-189.

Toide K, Man ZW, Asahi Y, Sato T, Nakayama N, Noma Y, Oka Y \& Shima K 1997 Glucose transporter levels in a male spontaneous non-insulindependent diabetes mellitus rat of the Otsuka Long-Evans Tokushima Fatty strain. Diabetes Research and Clinical Practice 38 151-160.

Ueda H, Ikegami H, Yamato E, Fu J, Fukuda M, Shen G, Kawaguchi Y, Takekawa K, Fujioka Y, Fujisawa T et al. 1995 The NSY mouse: a new animal model of spontaneous NIDDM with moderate obesity. Diabetologia 38 503-508.

Ueda H, Ikegami H, Kawaguchi Y, Fujisawa T, Nojima K, Babaya N, Yamada K, Shibata M, Yamato E \& Ogihara T 2000 Age-dependent changes in phenotypes and candidate gene analysis in a polygenic animal model of Type II diabetes mellitus; NSY mouse. Diabetologia 43 932-938.

Veroni MC, Proietto J \& Larkins RG 1991 Evolution of insulin resistance in New Zealand obese mice. Diabetes 40 1480-1487.

Watson RT \& Pessin JE 2006 Bridging the GAP between insulin signaling and GLUT4 translocation. Trends in Biochemical Sciences 31 215-222.

Weyer C, Bogardus C, Mott DM \& Pratley RE 1999 The natural history of insulin secretory dysfunction and insulin resistance in the pathogenesis of type 2 diabetes mellitus. Journal of Clinical Investigation 104 787-794.

Wild S, Roglic G, Green A, Sicree R \& King H 2004 Global prevalence of diabetes: estimates for the year 2000 and projections for 2030. Diabetes Care 27 1047-1053

\section{Received in final form 23 August 2006 \\ Accepted 30 August 2006 \\ Made available online as an Accepted Preprint 15 September 2006}

